\title{
A reevaluation of fluid flow, heat transfer and chemical reaction in catalyst filled tubes
}

\author{
Thomas Daszkowski and Gerhart Eigenberger \\ Institut für Chemische Verfahrenstechnik der Universität Stuttgart \\ Böblingerstrabe 72, D-7000 Stuttgart 1, Germany
}

\begin{abstract}
The problem of heat transfer through the wall in catalyst filled tubes with a tube to particle diameter ratio greater than $4 \div 5$ and industrial flow rates with and without chemical reaction has been reevaluated through detailed experiments and model calculations. The results confirm and extend findings of Vortmeyer et al. that reaction and heat transfer can only be modeled with independently determined parameters if the radial variation of the axial mass flow velocity is properly considered. In this case the wall heat transfer coefficient $h_{w}$ and the effective conductivity $\lambda_{r}$ are independent of lenght and have values about 20 to $40 \%$ lower than those obtained from available correlations that are based upon plug flow.
\end{abstract}

1. Introduction. Standard fixed-bed reactor models assume plug flow of the fluid pliase and describe the heat transfer through the wall with a mean radial conductivity $\lambda_{r}$ of the packed bed and a wall heat transfer coefficient $h_{w}$. It has been shown in many cases (Eigenberger and Ruppel, 1985; Hofmann, 1979; Paterson and Carberry, 1983) that these models are not able to represent experimental results quantitatively, if independently determined reaction kinetics and heat transfer parameters are used. There is increasing evidence that the main reason for this difficulty results from neglecting or insufficiently considering the radially varying flow profile (Delmas and Froment, 1988; Haidegger et al., 1989; Vortmeyer and Schuster, 1983). This paper summarizes results of a research project on the mutual influence of fluid flow, heat transfer through the wall and chemical reaction in catalyst filled tubes. The following points have been studied both experimentally and through detailed mathematical modeling:

- The radially and axially varying flow profile in catalyst filled tubes.

- Heat transfer through the wall without chemical reaction for different heat flow conditions and particle shapes.

- The axial and radial temperature and composition profiles for a catalytic oxidation reaction cooled through the wall for catalyst particles of different shape.

The main results obtained will be briefly discussed in the following. Details can be found in a thesis (Daszkowski, 1991) and will be published soon.

2. Fluid flow in randomly packed tubes. The fluid flow in randomly packed tubes is determined by local phenomena. Only after averaging in time and around a periphery, a regular flow structure becomes visible. Since gas flow measurements inside the packing are almost impossible without disturbing the flow, reliable flow measurements to date can only be obtained above or below the packing. Since the flow changes drastically while entering or leaving a packing, the measurements obtained outside do not represent the flow inside of the packed bed. Any flow modeling must therefore include the empty space above and below the packed bed (Fig. 1).

In this work the extended Brinkmann equation as proposed by Vortmeyer et al. (Haidegger et al., 1989; Vortmeyer and Schuster, 1983) has been used. It contains a momentum balance based upon a semi-empirical superposition of the Ergun equation for the pressure drop within the 
packing and a Navier-Stokes term to consider the non-slip condition at the tube wall as well as the empty inlet and outlet sections. Its advantage is the proper representation of the two limiting cases - packed bed with uniform porosity and the empty tube with laminar flow. The transition between these cases is obviously questionable.

Two momentum balances and the continuity equation determine the isothermal flow profile in the axial and radial coordinate. As an example only the axial momentum balance is given,

$$
\frac{\partial p}{\partial z}+\varepsilon f_{1} v_{z}+\varepsilon^{2} f_{2} v_{z}|v|+\rho_{g} v_{z}\left[\frac{\partial v_{z}}{\partial z}\right]+\rho_{g} v_{r}\left[\frac{\partial v_{z}}{\partial r}\right]-\mu\left[\frac{\partial^{2} v_{z}}{\partial z^{2}}\right]-\mu\left[\frac{\partial^{2} v_{z}}{\partial r^{2}}+\frac{1}{r} \frac{\partial v_{z}}{\partial r}\right]=0
$$

where the Ergun terms $f_{1}, f_{2}$ are strongly dependent upon the local porosity $\varepsilon(r, z)$. Contrary to previous work, the full variation of $\varepsilon=\varepsilon(r, z)$ in the simulation domain of Fig. 1 has been determined experimentally for tubes packed with spheres and used in the simulations. As a result, Fig. 2 shows the computed flow profile for the axial and the radial flow component for isothermal conditions.

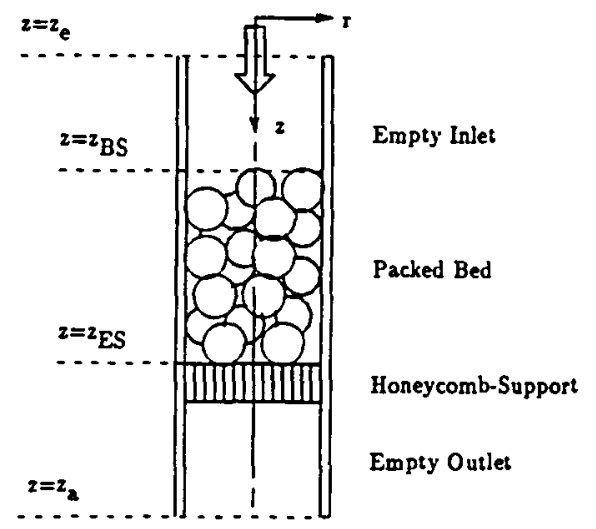

Figure 1: Simulation domain for flow modeling including the empty inlet and outlet section
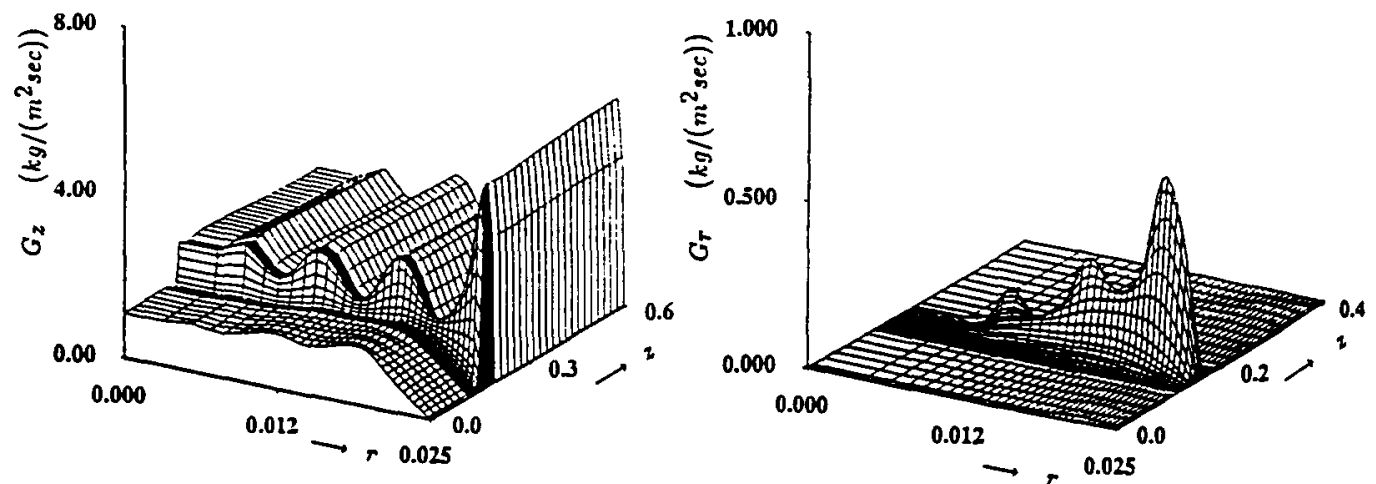

Figure 2: Computed flow profiles in the simulation domain. $G_{z}(z, r)$ : interstitial axial mass flow velocity, $G_{r}(r, z)$ : interstitial radial mass flow velocity.

A comparison between the measured and computed axial flow velocity $5 \mathrm{~mm}$ below the packing gives reasonable agreement. Fig. 3 shows some examples. These and other results lead to the following conclusions concerning fluid flow.

- The model used seems to represent the main features of fluid flow in packed beds reasonably well.

- The axial flow in the packed bed is characterized by a pronounced by-pass at the wall and a reduced mass flow in the centre.

- The build-up of the flow profile through a strong radial flow component mainly takes place in the first particle layer. Thereafter the axial mass flow profile stays constant, the radial component is zero.

- Contrary to the volumetric flow the mass flow profile is not much affected by radial tempe. rature gradients. 


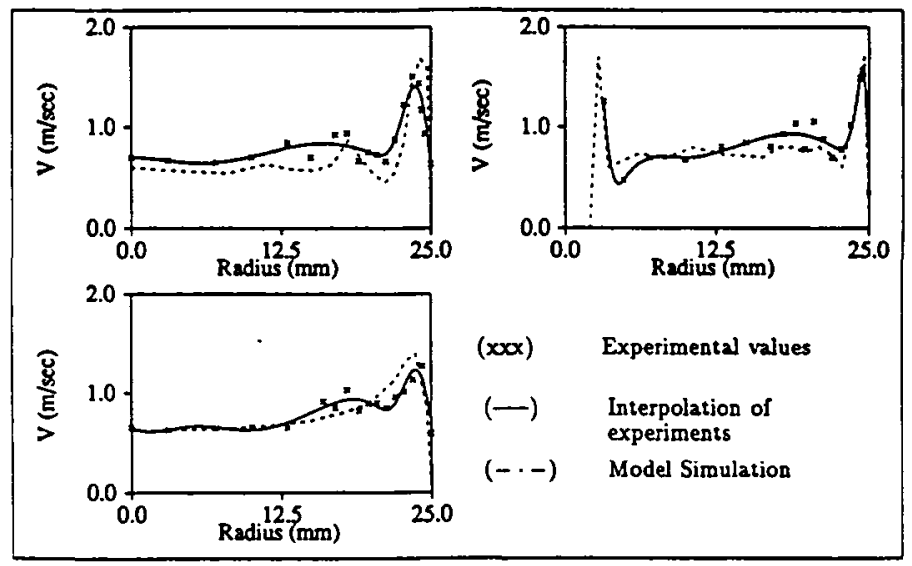

Figure 3: Axial velocity profiles $5 \mathrm{~mm}$ below the packing. Top left: spheres with $d_{p}=8 \mathrm{~mm}$, top right: spheres with $d_{p}=5 \mathrm{~mm}$ and central thermal well, bottom left: cylinders with $d_{p}=5 \mathrm{~mm}$ and a length of $\bar{l}=11 \mathrm{~mm}$.

3. Heat transfer in packed beds. Following the traditional approach, heat transfer was modeled by considering a mean radial conductivity $\lambda_{r}$ and a wall heat transfer coefficient $h_{w}$, which are assumed constant in axial $(z)$ and radial $(r)$ direction. The following pseudohomogeneus twodimensional energy balance with appropriate boundary conditions was added to model the heat transfer experiments.

$$
\left(\varepsilon \varrho_{g} c p_{g}+(1-\varepsilon) \varrho_{s} c p_{s}\right) \frac{\partial T}{\partial t}=-c p_{g} \mathrm{G}_{\mathbf{z}} \varepsilon \frac{\partial T}{\partial z}-c p_{g} \mathrm{G}_{\mathbf{r}} \varepsilon \frac{\partial T}{\partial r}+\lambda_{z} \frac{\partial^{2} T}{\partial z^{2}}+\lambda_{\mathbf{r}}\left[\frac{\partial^{2} T}{\partial r^{2}}+\frac{1}{r} \cdot \frac{\partial T}{\partial r}\right]
$$

The boundary condition at the wall $r=R$ is:

$$
\left.\frac{\partial T}{\partial r}\right|_{r=R}=\frac{\mathbf{h}_{\mathrm{w}}}{\lambda_{r}}\left(T_{w}-\left.T\right|_{r=R}\right)
$$

As shown above, the radial mass flow $G_{r}(z, r)$ can be neglected in most cases. Heat transfer experiments were conducted in an apparatus that allowed for an almost stepwise change of the wall temperature profile (Fig. 4, left). Care was taken that the entering gas temperature was constant over the radius and the thermocouples did not obstruct the flow. In all experiments the cold and the hot section were filled with the same packing so that the flow profile was established before the heating occured.

Comparison showed that both the conventional two-dimensional plug flow model and the detailed flow model were able to represent the measured radial temperature profiles at different positions equally well, provided the heat transfer parameters were properly adjusted to the respective model. Both parameters of the detailed flow model turned out to be about $20-40 \%$ lower than those of the plug flow model since in the detailed flow model the bulk of the fluid is flowing closer to the wall. It also turned out that the parameters of the plug flow model were length dependent, whereas the parameters of the detailed flow model did not show length effects. Assuming that the detailed model represents the real situation reasonably well, a number of simulation studies concerning the difference between heat transfer parameters derived for the two different models were performed. From these studies and the above mentioned experiments the following conclusions can be drawn:

- The plug flow model is not able to represent heat transfer experiments with values of the heat transfer coefficients that are independent of bed length and heating conditions, 
- in particular, different values of the heat transfer parameters are necessary in the plug flow model to describe heat transfer with constant wall temperature as compared to constant heat flux ( $h_{w}$ slightly increased, $\lambda_{r}$ decreased).

- Heat transfer parameters from experiments with constant wall temperature proved to be very sensitive to

- the assumed flow profile,

- the actual wall temperature profile,

- the temperature difference between wall and packing, which cannot be measured with sufficient accuracy if the packing has approached wall temperature.

This may explain the large spread of heat transfer parameters in the literature.

- Heat transfer parameters using the detailed flow model were the same for wall heating and wall cooling conditions, supporting the result of the flow studies that the mass flow profile was not significantly changed by the temperature profile in the range investigated $\left(20-300^{\circ} \mathrm{C}\right)$.

4. Reaction and heat transfer in packed beds. To provide detailed experimental results for a comparison with model calculations, a special laboratory fixed-bed reactor was designed (Fig. 4, right). It allowed for the measurement of radial temperature and concentration profiles at eight levels in the packed bed. The probes were installed in slices of honeycomb support between sections of the packed bed while careful packing minimized deviations in the flow, temperature and concentration profiles. In addition to temperature measurements the energy transferred to the wall in each of the packed bed sections was measured independently by a calorimetric technique.
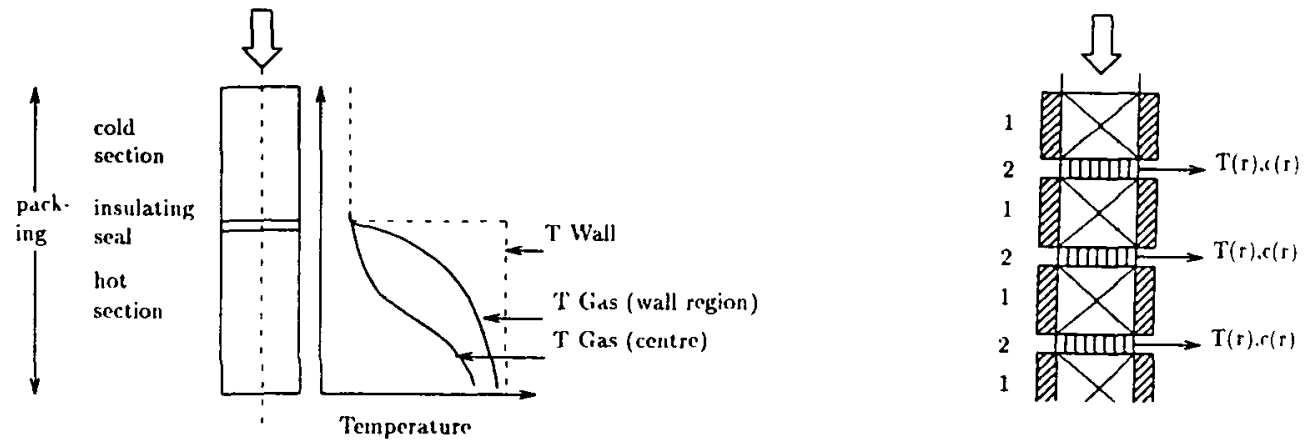

Figure 4: Left: Sketch of experimental set-up and temperature profiles duing wall heat transfer experiments, Right: Sketch of the laboratory reactor. Packed bed sections with constant wall temperature and heat flux measurement (1) and honeycomb slices for radial temperature and concentration measurements (2).

The total oxidation of propene on Pd supported catalysts of different geometry was used as a test reaction. The kinetics for the different catalysts were determined independently in a well mixed kinetic reactor. A comparison between experimental results and model calculations with no adjustable parameters using the heat transfer parameters determined in the above mentioned independent heat-up experiments gave the following results:

Only the detailed flow model was able to model experiments with different operating conditions reasonably well (Fig.5, 6, Table 1). The plug flow model gave a lower hot spot temperature and less conversion; the radial differences in the concentration profile were much less than in reality. The differences between the two models were largest in a small range of wall temperatures where the reaction was fast enough to produce a reasonable hot spot and conversion but not so fast to be completed between the first and the second measurement levels. In the latter case the temperature rose to almost the adiabatic value and the gas was cooled down subsequently as in a constant wall temperature experiment without reaction. 


\begin{tabular}{|l|l|l|l|}
\hline Heat flux (W) & Experiment & Detailed flow model & Plug flow model \\
\hline \hline Section2 & 15.1 & 14.2 & 14.66 \\
Section3 & 30.5 & 31.3 & 18.4 \\
Section4 & 29.2 & 31.1 & 29.2 \\
Section5 & 20.1 & 18.0 & 22.3 \\
Section6 & 11.1 & 9.0 & 11.3 \\
\hline Sum & 106.0 & 103.6 & 95.9 \\
\hline
\end{tabular}

Table 1: Comparison between measured and calculated heat flux in the reactor sections.

The difference between the two models can be easily explained qualitatively: compared to the plug flow model the detailed flow model has lower heat transfer parameters and a longer residence time in the tube center. Hence the reaction in the centre must lead to higher temperatures and conversions which drives the whole reaction to completion faster. Since the detailed model is able to describe the local profiles of temperature and conversion for a simple reaction reasonably well, it can be argued that its benefits will be even more pronounced in the modelling of temperature sensitive multistep reactions like partial oxidations or partial hydrogenations.

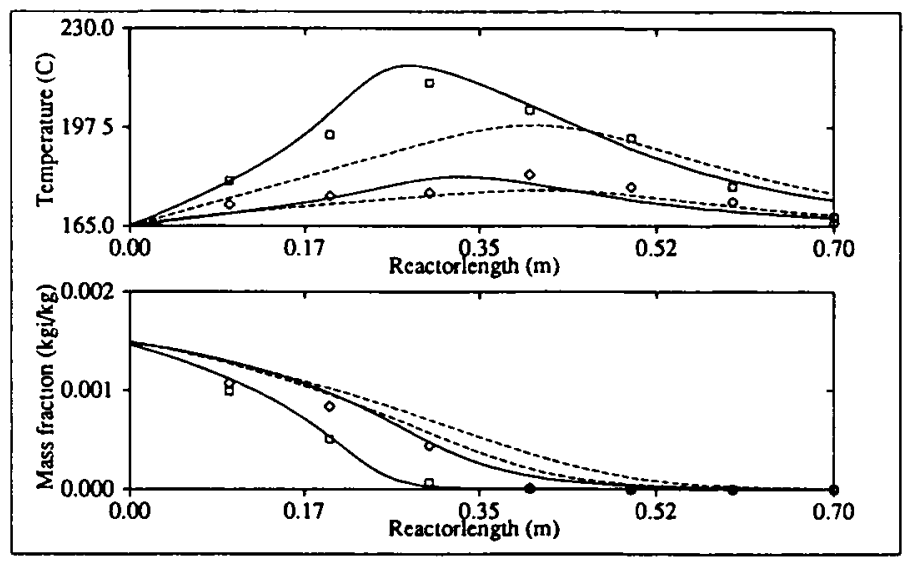

Figure 5: Measured (points) and calculated temperature and concentration profiles in the tube centre $(\square)$ and close to the wall $(\diamond),-$, detailed flow model and --- plug-flow model.

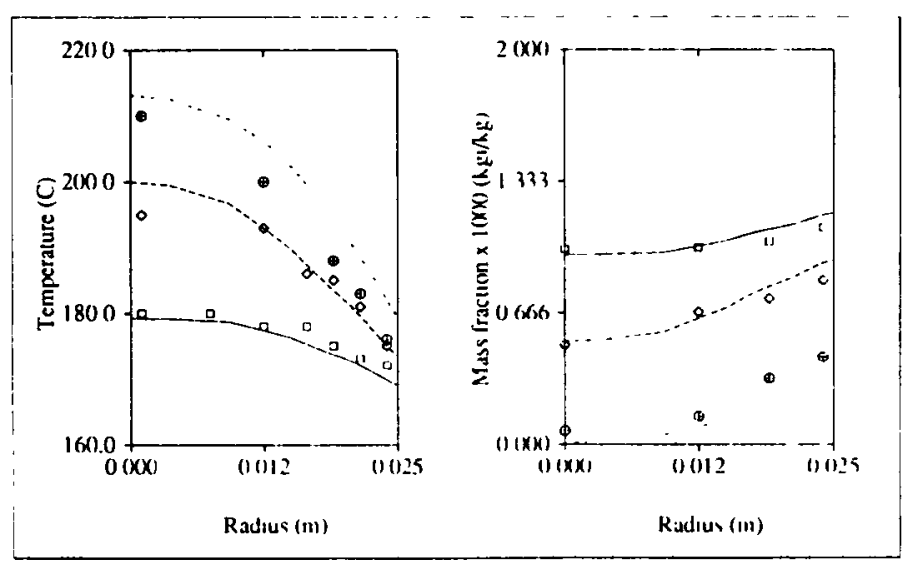

Figure 6: Measured and calculated temperature and concentration profiles at different positions over the radius. 
5. Conclusions It has been shown that heat transfer and reaction in catalyst filled tubes under industrial operating conditions can only be modeled with independently determined parameters if the radial variation of the axial mass flow component is properly considered. The results presented support and extend earlier work of Vortmeyer et al. (Vortmeyer and Schuster, 1983; Haidegger et al., 1989). They differ somewhat in the underlying velocity profile, which in this work was based upon measurements with different pellet geometries. It was shown that the two traditional heat transfer parameters, the wall heat transfer coefficient $h_{w}$, which is constant over $z$ and the effective radial conductivity $\lambda_{r}$, which can be taken constant in $z$ and $r$, describe heat transfer with and without reaction reasonably well, if the detailed flow model is used. The values of both parameters however differ from those found in literature. Therefore a future task will be to determine new correlations for $\lambda_{r}$ and $h_{w}$ together with an appropriate flow description for common catalyst shapes and operating conditions.

The model simulations presented required about 30 radial and 100 axial grid points which leads to excessive computation times for a single steady state solution. A simplified model using only two radial grid points was therefore derived (Daszkowski, 1991). It is based upon the detailed flow profile and usually gives a good agreement with the full model with short computation times. Details will be communicated in a seperate contribution.

Acknowledgements- Support of this work through the Bundesminister für Forschung und Technologie and, for the part of the modeling work, through the Volkswagen-Stiftung is gratefully acknowledged.

\begin{tabular}{lllll} 
Notation & & \multicolumn{2}{c}{ Indices } \\
$c p$ & heat capacity & $J /(k g K)$ & $g$ & gas \\
$G$ & interstitial mass flow & $k g /\left(m^{2} s e c\right)$ & $r$ & radial coordinate \\
$h_{w}$ & wall heat transfer coefficient & $W /\left(m^{2} K\right)$ & $s$ & solid \\
$p$ & pressure & $b a r$ & $z$ & axial coordinate \\
$t$ & time & $s e c$ & & \\
$T$ & temperature & $K$ & & \\
$v$ & interstitial velocity & $\mathrm{m} / \mathrm{sec}$ & & \\
& & & \\
$\rho$ & density & $\mathrm{kg} / \mathrm{m}^{3}$ & & \\
$\mu$ & viscosity & $\mathrm{kg} /(\mathrm{msec})$ & \\
$\lambda$ & effective conductivity & $W /(\mathrm{mK})$ & & \\
$\varepsilon$ & void fraction & &
\end{tabular}

\section{References}

T. Daszkowski. Strömung, Stoff- und Wärmetransport in schüttungsgefüllten Rohren. Dissertation Universität Stuttgart, 1991.

H. Delmas and G. Froment. A simulation model accounting for structural radial nonuniformities in fixed bed reactors. Chemical Engineering Science, pages 2281-2287, 1988.

G. Eigenberger and W. Ruppel. Problems of mathematical modelling of industrial fixed-bed reactors Ger. Chem. Eng. 9, pages 74-83, 1986.

D.Haidegger, E. Vortmeyer and P. Wagner. Simultane Lösung von Energie-, Stoff- und Impulsgleichung für wandgekühlte chemische Festbettreaktoren. Chemie Ingenieur Technik 61, pages 647-650, 1989.

H. Hofmann. Fortschritte bei der Modellierung von Festbettreaktoren. Chemre Ingenteur Technik 51, pages 267-265, 1979.

W. Paterson and J. Carberry The heat transfer problem. Chemical Engineering Scrence 98, pages 175-180, 1983.

D. Vortmeyer and J. Schuster. Evaluation of steady flow profiles in rectangular and circular packed beds by a variational method. Chemical Engineering Science 98, pages 1691-1699, 1983. 